\title{
Evaluación de actitudes sociales en estudiantes de primer ingreso a psicología
}

María Inés Gómez del Campo del Paso ${ }^{1}$ e Ithzel Liliana Fernández Montaño ${ }^{1}$

\section{Resumen}

El objetivo de la presente investigación es evaluar las habilidades sociales de los estudiantes de nuevo ingreso a la Licenciatura en Psicología de una universidad pública en el estado de Michoacán, a través de la Escala de Actitudes y Estrategias Cognitivas y Sociales (AECS) (Moraleda, González y García-Gallo, 2004), con el fin de diseñar un programa de seguimiento longitudinal que permita, a través de una serie de talleres vivenciales, desarrollar la empatía, la colaboración y el liderazgo.

En este primer momento se presentan los resultados de la aplicación del test AECS a un grupo de 54 estudiantes, así como la comparación por género y las correlaciones entre las distintas actitudes, encontrando que no existen diferencias entre los grupos; sin embargo, las medias grupales se encuentran por abajo de lo esperado para su grupo de edad en las tres actitudes que se pretenden evaluar, lo que hace indispensable la elaboración de un programa de intervención para promover el desarrollo de estas actitudes.

1 Universidad Michoacana de San Nicolás de Hidalgo. 
Palabras clave: actitudes sociales, universitarios, psicología, colaboración, liderazgo.

\section{Introducción}

En la edad adulta emergente, Carstensen (1992) menciona que las interacciones sociales se vuelven muy funcionales. La entrada a la edad adulta es un momento en la vida donde gran cantidad de información se puede obtener a partir de la interacción social casual. Además, en este momento se consolidan las amistades cercanas, y los compañeros para toda la vida se eligen de una amplia gama de relaciones posibles. Después de los 30 años, hay una marcada reducción en la creación de nuevas relaciones sociales y la persona se centra en el mantenimiento de las relaciones ya adquiridas.

Berger y Thompson (2001) concuerdan al explicar que durante esta etapa, la intimidad adquiere mayor importancia, por lo tanto, el adulto emergente adopta diversos roles. Cada relación íntima tiene que ir de la atracción inicial hasta llegar a un compromiso; cada rol implica que el adulto emergente ofrezca algo de sí mismo, al mostrarse más auténtico y vulnerable con los demás adquiere una mayor comprensión de sí mismo, lo que evita el aislamiento. Las principales formas de intimidad en esta etapa son las amistades íntimas y el emparejamiento sexual.

Erikson (1993) explica que existe un problema en las relaciones interpersonales cuando la persona se permite a sí misma la fusión con un grupo para adquirir ciertos rasgos de identidad. Sin embargo, si el joven consigue relacionarse con el grupo conservando su individualidad, adquiere un sentido de identidad yoica a partir del cual podrá relacionarse íntimamente con otras personas y vivir de acuerdo con los estándares de la sociedad. A pesar de sus imperfecciones, entonces, la persona encontrará un lugar dentro de la sociedad que le permita contribuir a la estabilidad y el desarrollo de ésta.

Erikson (1993) establece que cuando el joven no es capaz de lograr la intimidad, se distancia y se aísla, considerando la presencia de los demás como una amenaza para su seguridad 
personal. Por lo tanto, evitará cualquier relación que requiera de cercanía emocional. Si los jóvenes adultos no pueden establecer compromisos personales profundos con los demás, quizá se mantengan aislados, evitando en lo posible las relaciones.

Por lo que es muy importante que durante la adultez emergente, el joven adquiera las actitudes sociales que le permitirán relacionarse de una manera adecuada con los demás y propiciarán su eficaz inserción en el campo profesional.

Tener habilidades sociales es fundamental para los psicólogos en cualquier área en la que se desenvuelvan, sea clínica, social, laboral o educativa, porque además del trabajo que se realiza con las personas, su actitud es elemental en el modelamiento de las mismas.

En la experiencia como docentes, se ha observado que algunos estudiantes no tienen un buen desarrollo de sus actitudes sociales, ya que muestran en las clases o en las prácticas realizadas: falta de empatía, falta de asertividad, dificultades para trabajar en equipo, para negociar o para tomar iniciativas, entre otras, lo que podría implicar dificultades en su posterior desempeño profesional.

Según Ballester y Gil (2009), una persona que es hábil socialmente se caracteriza por saber expresar lo que siente, necesita y le interesa de una forma tranquila, minimizando así la probabilidad de presentarse futuros problemas en las relaciones, ya que los demás saben acerca de lo que es importante para él.

Para Moraleda, González y García (2004), las habilidades sociales son variables que facilitan o dificultan la adaptación social al medio en el que viven los jóvenes.

De acuerdo con Díaz-Barriga y Hernández (2010: 2), actualmente se espera que el estudiante, como resultado del paso por las instituciones educativas, "aprenda a aprender, a colaborar, a comportarse de forma ética, responsable y solidaria, a resolver problemas, a pensar y recrear el conocimiento".

La capacidad para establecer relaciones interpersonales o competencia social (Trianes y Fernández-Figares, 2010) supone la adquisición de habilidades básicas de interacción y modos de comunicación no verbal; poseer complejas habilidades y estrategias cognitivas y afectivas de regulación de emociones, de representación del punto de vista del otro, conocimiento social 
de normas y valores y estilos de procesamiento de información, reflexivos y apropiados y, por último, poseer autopercepciones de éxito y eficacia en la resolución de conflictos durante una convivencia cotidiana. Además, presenta complejas relaciones con el desarrollo cognitivo, social y moral, ya que algunos componentes importantes de la competencia social se van adquiriendo lentamente y llegan a poseerse plenamente en la adolescencia, como por ejemplo, la consideración de necesidades del otro, la superación del egoísmo infantil, el manejo de las propias emociones o la conciencia moral como guía de la conducta.

Es decir, los estudiantes acuden a las universidades para obtener una serie de conocimientos, habilidades intelectuales y técnicas prácticas que les servirán para desarrollarse en el ámbito profesional. Pero además, deben estar capacitados para comunicarse eficazmente, coordinar equipos, tomar decisiones, resolver conflictos interpersonales, negociar y acordar, independientemente de la disciplina en la que se estén preparando (Greco y Prendes, 2008).

Existen diversas investigaciones en el campo de la psicología que enfatizan la importancia de las actitudes sociales para el adecuado desempeño de los estudiantes universitarios. Se ha encontrado que aquellos estudiantes que tienen más desarrolladas estas actitudes, tienen mejores relaciones interpersonales en la universidad y por lo tanto mayores posibilidades de concluir la carrera con éxito.

Como punto de partida para el desarrollo de estas actitudes, es necesario medir el nivel en el que los estudiantes las poseen, por lo que el objetivo de la presente investigación es evaluar las actitudes sociales de los estudiantes de nuevo ingreso a la Licenciatura en Psicología de una universidad pública en el estado de Michoacán, a través de la Escala de Actitudes y Estrategias Cognitivas y Sociales (AECS) (Moraleda, González y García-Gallo, 2004), con el fin de diseñar un programa de seguimiento longitudinal que permita, a través de una serie de talleres vivenciales, desarrollar la empatía, la colaboración y el liderazgo. 


\section{Método}

Este es un estudio exploratorio de detección de necesidades para planear una intervención posterior.

Participantes. 54 estudiantes de segundo semestre de la Licenciatura en Psicología de una universidad pública que se prestaron voluntariamente para participar en la investigación, de edades entre 17 y 19 años. 20 hombres y 34 mujeres.

Instrumento. Escala de Actitudes y Estrategias Cognitivas y Sociales (AECS) (Moraleda, González y García-Gallo, 2004). La cual consta de 137 reactivos que se responden a través de una escala de Likert de 7 opciones.

La propuesta de competencia social del AECs se fundamenta principalmente en que el éxito o fracaso de los jóvenes en sus interacciones sociales, depende de la presencia de ellos en ciertas variables actitudinales y cognitivas consistentes y estables a través del tiempo. Evalúa actitudes sociales y pensamiento social.

Las escalas de actitudes sociales son: conformidad con lo que es socialmente correcto, sensibilidad social, ayuda y colaboración, seguridad y firmeza en la interacción, liderazgo prosocial, agresividad -terquedad, dominancia, apatía-, retraimiento, ansiedad -timidez.

Las escalas de pensamiento social son: impulsividad -reflexividad, independencia-, dependencia de campo, convergencia-divergencia-, percepción y expectativas negativas sobre la relación social, percepción positiva del sujeto del modo de ejercer sus padres la autoridad en el hogar, percepción negativa del sujeto de la calidad de aceptación y acogida que recibe de sus padres, dificultad en la observación y retención de la información relevante sobre situaciones sociales, dificultad en la búsqueda de soluciones alternativas para resolver problemas sociales, dificultad para anticipar y comprender las consecuencias que posiblemente seguirán de los comportamientos sociales y dificultad para elegir los medios adecuados para los fines que se persiguen en el comportamiento social. 
Las escalas de actitud social que se consideraron más pertinentes por su relación con el perfil de ingreso y egreso deseables de la Facultad de Psicología fueron:

Sensibilidad social. Evalúa la tendencia a sintonizar con los sentimientos ajenos. La disposición a admitir en los demás modos de ser distintos de los propios; a valorar a los otros; a tener una imagen positiva de ellos. Confiabilidad: coeficiente alfa de 0.81 .

Ayuda y colaboración. Mide la tendencia a compartir con los demás lo propio, a estimular su rendimiento, a reforzarlos; a participar y colaborar en el trabajo común; a construir soluciones por consenso. Confiabilidad: coeficiente alfa de 0.88 . Seguridad y firmeza en la interacción. Analiza la confianza en las propias posibilidades para conseguir los objetivos de la interacción; la firmeza en la defensa de los propios derechos y en la expresión de las quejas; la tendencia a afrontar los problemas y a no evitarlos. Confiabilidad: coeficiente alfa de 0.88 . Liderazgo prosocial. Esta escala aprecia la tendencia a dar ideas en el grupo; a aunar a sus miembros en torno a objetivos comunes; a tomar la iniciativa; a planificar actividades con espíritu de servicio. Confiabilidad: coeficiente alfa de 0.75 .

Procedimiento. Se invitó a los estudiantes de segundo semestre a participar en la investigación. Se aplicó el instrumento a los 54 estudiantes que acudieron. Posteriormente, se realizó el análisis de los datos para obtener los estadísticos descriptivos de cada una de las escalas y se aplicó la correlación de Pearson para obtener las relaciones entre las distintas escalas, esto a través del programa estadístico spss versión 15. Después, los estudiantes participaron en un taller sobre "Habilidades para la vida".

\section{Resultados}

A continuación, se presentan las tablas que muestran los resultados obtenidos en la aplicación de las escalas. La primera de ellas muestra las medias por sexo en cada una de las actitudes. La segunda presenta las correlaciones entre las diferentes actitudes. 
Tabla 1. Datos estadísticos del grupo

\begin{tabular}{llll}
\hline \multicolumn{1}{c}{ Escala } & Sexo & N & Media \\
\hline Sensibilidad social & Hombre & 20 & 50.25 \\
\cline { 2 - 4 } & Mujer & 34 & 37.12 \\
\hline \multirow{2}{*}{ Ayuda y colaboración } & Hombre & 20 & 56.15 \\
\cline { 2 - 4 } & Mujer & 34 & 44.09 \\
\hline Seguridad y firmeza & Hombre & 20 & 45.90 \\
\cline { 2 - 4 } & Mujer & 34 & 30.12 \\
\hline \multirow{2}{*}{ Liderazgo prosocial } & Hombre & 20 & 57.55 \\
\cline { 2 - 4 } & Mujer & 34 & 49.97 \\
\hline
\end{tabular}

En la Tabla 1 se puede observar que la media de los hombres en sensibilidad social, ayuda y colaboración y liderazgo prosocial es más alta que la de las mujeres. Sin embargo, las diferencias no son significativas. También se observa que la puntuación en seguridad y firmeza es la más baja en ambos sexos, mientras que liderazgo prosocial es la más alta; aun la más alta está en un percentil entre 50 y 60, y lo deseable es que estuviera en 70 o más. 
Tabla 2. Correlaciones entre las escalas

\begin{tabular}{|c|c|c|c|c|}
\hline & & $\begin{array}{l}\text { Sensibilidad } \\
\text { social }\end{array}$ & $\begin{array}{c}\text { Ayuda } \\
\text { y colaboración }\end{array}$ & $\begin{array}{c}\text { Seguridad } \\
\text { y firmeza }\end{array}$ \\
\hline \multirow{2}{*}{ Sensibilidad social } & Correlación de Pearson & & & \\
\hline & Sig. (bilateral) & & & \\
\hline \multirow{2}{*}{ Ayuda y colaboración } & Correlación de Pearson & $0.416^{\star *}$ & & \\
\hline & Sig. (bilateral) & 0.002 & & \\
\hline \multirow{2}{*}{ Seguridad y firmeza } & Correlación de Pearson & $0.331^{*}$ & $0.512^{* *}$ & \\
\hline & Sig. (bilateral) & 0.014 & 0.000 & \\
\hline \multirow{2}{*}{ Liderazgo prosocial } & Correlación de Pearson & 0.207 & $0.370^{* x}$ & $0.378^{* *}$ \\
\hline & Sig. (bilateral) & 0.129 & 0.005 & 0.004 \\
\hline
\end{tabular}

**La correlación es significativa al nivel 0,01 (bilateral).

*La correlación es significante al nivel 0,05 (bilateral).

c. $\mathrm{N}$ por lista $=55$.

La Tabla 2 muestra la correlación positiva más alta entre ayuda y colaboración y seguridad y firmeza; también muestra correlación alta entre ayuda y colaboración y sensibilidad social; en tercer lugar, podemos observar una correlación positiva de liderazgo prosocial con seguridad y firmeza y con ayuda y colaboración. Mientras que no existe correlación entre liderazgo prosocial y sensibilidad social. Cabe mencionar que las correlaciones muestran un nivel de significancia superior a 0.01 .

\section{Conclusiones y discusión}

Es importante comentar que las actitudes evaluadas no tienen el nivel de desarrollo que se esperaría para el perfil de ingreso de los estudiantes de psicología, y no necesariamente se van a desarrollar por sí mismas durante la carrera, por lo que se hace necesario proponer estrategias para incrementarlas.

Pérez y colaboradores (2010), en una investigación que se llevó a cabo en España, encontraron que en los estudiantes universitarios las competencias emocionales que tienen una re- 
lación muy directa con las actitudes sociales que se están evaluando, se vinculan con niveles óptimos de desempeño en los ámbitos personal, educativo, profesional y en el afrontamiento exitoso de los retos en la vida diaria.

Por lo tanto, se considera pertinente realizar acciones tendientes a incrementar estas actitudes en nuestros estudiantes para favorecer mejores niveles de desempeño en las áreas que mencionan los autores.

Esta necesidad se corrobora también con lo expresado por Extremera (2005), quien encontró que aquellos estudiantes con niveles elevados de manejo emocional muestran mayores niveles de intimidad y afecto hacia sus amigos. Igualmente, aquellos alumnos con altas puntuaciones en manejo emocional muestran mayor implicación empática y una mayor toma de perspectiva, así como menores niveles de malestar personal. Sugiriendo la importancia de un buen meta-conocimiento de las propias emociones como base para comprender las emociones de los demás, ya que las actitudes sociales evaluadas impactan directamente en el manejo emocional y en las relaciones con los demás.

Los resultados de la presente investigación muestran que el bajo puntaje en las actitudes de sensibilidad social, ayuda y colaboración, seguridad y firmeza y liderazgo prosocial, requiere estrategias para su desarrollo durante la formación como psicólogos, con el fin de promover un mejor desempeño durante sus estudios universitarios y en su vida profesional.

A partir de las correlaciones, se puede observar que las personas que muestran alta puntuación en ayuda y colaboración también tendrán desarrollada la seguridad y firmeza y la sensibilidad social. Mientras que el ser sensible socialmente no garantiza que la persona sea un buen líder.

Esto implica que los estudiantes que muestran tendencia a sintonizar con los sentimientos ajenos y disposición a aceptar y valorar a los otros, también son más firmes en la defensa de sus propios derechos y en la tendencia a afrontar los problemas y no evitarlos, y buscan participar y colaborar en el trabajo común, así como en la construcción de soluciones por consenso. Esto se relaciona con lo que establecen Trianes y Fernández-Figares (2010), respecto a que la intimidad se asocia a una relación de 
colaboración en la que los amigos se implican en actividades mutuas, con confianza uno en otro y sentimientos de satisfacción personal con la relación.

Por otro lado, el hecho de ser capaz de empatizar con los demás o ser socialmente sensible, no garantiza que la persona esté dispuesta a tomar la iniciativa frente a un grupo o a planificar actividades para el bien común. Por lo que será importante desarrollar en los estudiantes el liderazgo prosocial, ya que éste favorecerá también el incremento de las actitudes de ayuda y colaboración.

Esto concuerda con lo encontrado por Pérez, Filella y Sodevila (2010), quienes realizaron una investigación con estudiantes universitarios en España, encontrando una correlación positiva entre las dimensiones de la competencia emocional (evaluadas con el Cuestionario de Desarrollo Emocional para Adultos) y las habilidades sociales (medidas a través de la Escala de Habilidades Sociales), en donde concluyen que aquellos estudiantes universitarios con altas puntuaciones en competencia emocional, muy probablemente también obtendrán puntajes altos en habilidades sociales.

Sin embargo, estas competencias no se encuentran muy desarrolladas al momento de ingresar a la universidad, por lo que es importante reconocer e impulsar la incorporación de actividades para el desarrollo de las competencias socio-emocionales en el contexto de la enseñanza universitaria.

Entonces, para que el estudiante de psicología desarrolle sus actitudes sociales, deberá contar con espacios de diálogo personal que favorezcan el desarrollo de la escucha y la empatía, y que pongan énfasis en la congruencia interna de la comunicación, como las que se propician a través de ejercicios grupales de escucha, diálogo, toma de decisiones y resolución de problemas en grupo.

Una de las formas de que las universidades proporcionen a los jóvenes este desarrollo integral, es la que propone la Psicología Humanista a través de los talleres vivenciales. Ya que éstos son trabajos grupales que por sus características de duración, tamaño del grupo e intensidad emocional de los ejercicios, pueden incorporarse con facilidad a los programas de las ma- 
terias, y su objetivo primordial es mejorar las relaciones interpersonales de los participantes a través de diversos ejercicios estructurados.

Por lo anterior descrito, se concluye que es pertinente una intervención grupal con enfoque humanista a partir de ejercicios vivenciales, ya que la experiencia con otros participantes y las actitudes promotoras del crecimiento de parte del facilitador potencializarán las actitudes sociales de los estudiantes y se verán favorecidos tanto en el área personal como profesional.

\section{Referencias}

Ballester, R. y Gil, D. (2009). Habilidades sociales. España: Síntesis.

Berger, J. y Thompson, L. (2001). Psicología del desarrollo: adultez y vejez. España: Editorial médica panamericana.

Carstensen, L. (1992). Social and emotional patterns in adulthood: Support for socioemotional selectivity theory. Psychology and Aging, 7(3), 331-338.

Díaz-Barriga, F. y Hernández, G. (2010). Estrategias docentes para un aprendizaje significativo. Una interpretación constructivista. México: McGraw-Hill.

Erikson, E. H. (1993). Infancia y sociedad. Buenos Aires: Lumen-Hormé.

Extremera, P. N. (2005). Inteligencia emocional, calidad en las relaciones interpersonales y empatía en estudiantes universitarios. Clínica y Salud. 15(2), 117-137.

Greco, M. y Prendes, C. (2008) Competencias sociales en la universidad, visión de docentes y alumnos. En Cátedras electrónicas Universidad Nacional del Litoral. Disponible en: www.fcv.unl.edu.ar/archivos/grado/catedras.

Ibarra Mendivil, J. L.(2003) La universidad necesaria. En REDIE Revista Electrónica de Investigación Educativa, (5).

Moraleda, M., González, A. y García-Gallo, J. (2004). AEcs. Actitudes y estrategias cognitivas sociales. Madrid: TEA.

Pérez, E. N., Filella, G. G. y Sodevila, B. A. (2010). Competencia emocional y habilidades sociales en estudiantes uni- 
versitarios. Revista Electrónica de Motivación y Emoción, 13(34), 3-11.

Trianes Torres, M. V. y Fernández-Figares Morales, C. (2010). Aprender a ser personas y a convivir: un programa para secundaria (2a. ed.). España: Desclée de Brouwer. 